\title{
Severe Bradycardia during carotid-femoral Pulse Wave Velocity Measurement.
}

\author{
Denis Souza ${ }^{1}$ and Sebastiao Rodrigues Ferreira-Filho ${ }^{2}$ \\ ${ }^{1}$ Federal University of Uberlandia \\ ${ }^{2}$ Federal University of Uberlândia - Umuarama Campus
}

April 28, 2020

\begin{abstract}
This case showed that an elderly patient presented severe bradycardia concomitantly with consciousness lost, during the measuring of carotid-femoral Pulse Wave Velocity (c-fPWV). We propose that a single measure of c-f PWV is enough for older people.
\end{abstract}

Title:

Severe Bradycardia during carotid-femoral Pulse Wave Velocity Measurement.

Authors: Denis Fabiano Souza; Sebastião R Ferreira-Filho (1)

1: Internal Medicine Unit, Federal University of Uberlandia, MG; Brazil.

Corresponding Author's email address: sebahferreira@gmail.com

Key Words:

Geriatric Medicine, Nephrology.

Key Clinical Message: This case showed that an elderly patient presented severe bradycardia concomitantly with consciousness lost, during the measuring of carotid-femoral Pulse Wave Velocity (c-fPWV). We propose that a single measure of c-f PWV is enough for older people.

Introduction:

The statement commissioned by the American Heart Association, which addresses the nomenclature, methodologies, utility, limitations, and gaps in knowledge for the arterial stiffness measurements, has a special section about how to perform applanation tonometry [1]. In this statement, there is a special section with many recommendations for measuring arterial stiffness, including a suggestion that the measurements should be performed in duplicate after a minimum of 10 minutes of rest. The authors also recommend that pulse wave velocity (PWV) measurements should be repeated a third time if the difference between the two initial measurements is $>0.5 \mathrm{~m} / \mathrm{s}$. The statement indicates that operators should be familiar with the equipment and have been trained in the techniques.

The apllanation tonometry (AT) to measure carotid-femoral PWV, can be a barrier for many kinds of populations, such as elderly and very older people. This special population often does not tolerate remaining in bed in a supine position without raising the head of the bed due to respiratory or joint problems. Additionally, the repeated compressions to the cervical area by the tonometer may stimulate the baroreceptors and promote unnecessary, and even dangerous, heart rate reductions. 
After the case that will be reported below, we hypothesized that a single measurement of the carotid-femoral PWV (cf-PWV) obtained using AT, performed by an experienced nurse, could be very close to the mean value obtained by three consecutive measures.

Case Presentation

A 67-year-old man patient who, during slight tonometer compression of the carotid artery to measure the carotid-femoral PWV, presented with severe bradycardia and loss of consciousness, necessitating the interruption of the exam (Figure 1). His medical history was unremarkable; physical examination and results of laboratory exams were in the normal range. Subsequently, the patient was referred for a duplex carotid scan. During the duplex carotid scan, the patient again presented bradycardia, necessitating the interruption of the examination. Although the exam was partially performed, no anatomical changes in the carotids were observed.

Discussion:

We performed carotid-femoral pulse wave velocity (cf-PWV) in the thirty-eight elderly patients with three sequential measurements made by the same trained observer using the SphygmoCor device (AtCor Medical, Sydney, Australia). The subjects were placed in a supine position for at least 10 minutes, and we performed three consecutive cf-PWV measurements.

The subjects had an average age of $67 \pm 5.8$ years, with an average $\mathrm{BP}$ of $131 / 75 \pm 21 / 11 \mathrm{mmHg}$, heart rate of $72 \pm 11 \mathrm{bpm}$, and cf-PWV of $7.8 \pm 2.0 \mathrm{~m} / \mathrm{s}$. Three successful measurements were obtained in all subjects. We quantified the limits of agreement between the measurements using Bland-Altman plots[2]. The average difference (SD) was $0.09(1.98) \mathrm{m} / \mathrm{s}, 0.14(2.01) \mathrm{m} / \mathrm{s}$, and $0.06(1.98) \mathrm{m} / \mathrm{s}$ between the first and second, first and third, and second and third measurements, respectively. Therefore, the $95 \%$ limits of agreement were $3.88 \mathrm{~m} / \mathrm{s}, 3.94 \mathrm{~m} / \mathrm{s}$ and $3.88 \mathrm{~m} / \mathrm{s}$, respectively. The average range of variation between measurements within individual subjects was $0.39 \mathrm{~m} / \mathrm{s}$ (range 0 to $1.7 \mathrm{~m} / \mathrm{s}$ ). Only 3 subjects had a range of variation $>1$ $\mathrm{m} / \mathrm{s}$, and 26 subjects had a range $<0.5 \mathrm{~m} / \mathrm{s}$. The Lin [3] concordance coefficient for the 38 sets of triplicate measurements (114 comparisons) was 0.978 (95\% limits 0.968-0.985), indicating a "substantial" agreement between the measurements (Figure 2).

Conclusion:

In conclusion, sequential measurements of cf-PWV showed almost perfect agreement (arbitrarily defined when Lin's coefficient is $>0.99$ ). We conclude that a single cf-PWV measurement provides enough precision in elderly hypertensive patients. This finding has particular relevance to the practicability of implementing AT in clinical practice, mainly in special populations such as older people. A single measurement would avoid multiple compressions in the cervical region and reduce the chances of undesirable effects on heart rate for the measurements of cf-PWV.

Author contribution :

Author 1: Execution, writing and protocol design.

Author 2: Execution, writing and protocol design.

References:

1. Townsend RR, Wilkinson IB, Schiffrin EL, Avolio AP, Chirinos JA, Cockcroft JR, et al. Recommendations for Improving and Standardizing Vascular Research on Arterial Stiffness: A Scientific Statement From the American Heart Association. Hypertension 2015; 66 (3):698-722.

2. Bland JM, Altman DG. Statistical methods for assessing agreement between two methods of clinical measurement. Lancet 1986; 1 (8476):307-310.

3. Lin LI. A concordance correlation coefficient to evaluate reproducibility. Biometrics 1989; 45 (1):255-268.

Legends: 
Figure 1: A 67-year-old man, BP: 140/90 mmHg, had a reduction in HR during applanation tonometry for measuring carotid-femoral pulse wave velocity (c-fPWV).

Figure 2: The concordance correlation coefficient (Lin's test) determines how far the observed data deviate from the line of perfect concordance (i.e., the line at 45 degrees on a square scatter plot).

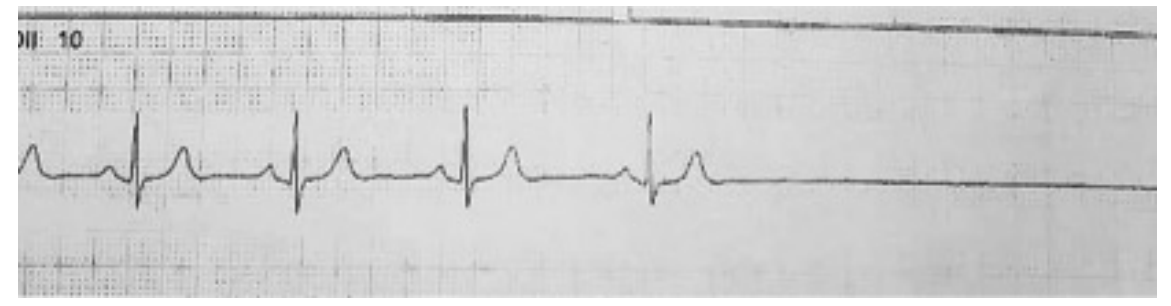




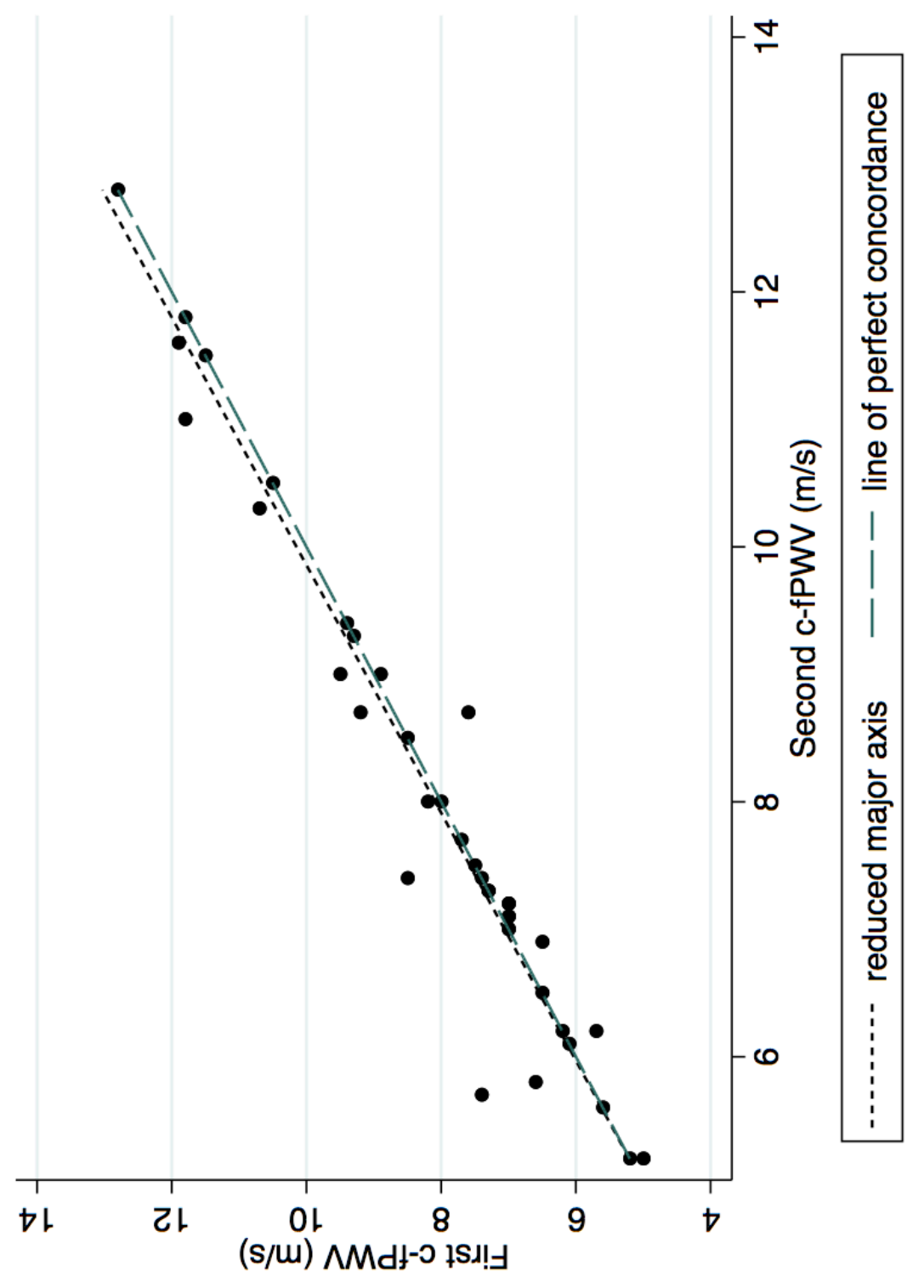

